\title{
Indicador de alerta temprana aplicada a empresas que conforman el índice de precios y cotizaciones en la Bolsa Mexicana de Valores
}

\author{
José Gerardo De la Vega Meneses ${ }^{1}$
}

Recibido: 3 de mayo de 2016

Aprobado: 17 de junio de 2016

\section{De la Vega, J. (2016). Indicador de alerta temprana aplicada a empresas que conforman el índice de precios y cotizaciones en la Bolsa Mexicana de Valores. Activos, 26, 155-181.}

Clasificación JEL: G00, G39

\section{Resumen}

Diversas empresas han incurrido en bancarrota en el pasado y muchas podrían incidir en dicha situación en el futuro. Esto puede ser atribuido a los efectos combinados de una mayor competitividad en los mercados y a un excesivo apalancamiento financiero. Estos factores resultan más relevantes cuando van acompañados de crisis económicas o problemas ajenos a las empresas y que afectan económicamente a los países donde se desarrollan sus actividades. La probabilidad de supervivencia de las empresas puede inferirse utilizando análisis de estados financieros. Un modelo basado en estos análisis y que ha resultado verosímil en la predicción de bancarrota

1 Universidad Popular Autónoma del Estado de Puebla. 21 sur 1103, Colonia Santiago, Puebla, México, C.P. 72160. Tel.: (222) 22994 00, ext. 7757; fax: (222) 232 52 51. Correo electrónico: josegerardo.delavega@upaep.mx 
de las empresas dos años antes de que suceda es el modelo del marcador Z, de Edward Altman. El marcador Z, el cual se aplica preferentemente a empresas del sector manufacturero y que cotizan en bolsas de valores, fue utilizado en el presente estudio para estimar la probabilidad de bancarrota de las empresas que componen el índice de precios y cotizaciones de la Bolsa Mexicana de Valores, al cierre del año 2015.

\title{
Palabras claves
}

Marcador Z, análisis financiero, Bolsa Mexicana de Valores.

De la Vega, J. (2016). Early warning indicator applied to enterprises that make up the prices and quotation index of Mexican Stock Exchange. Activos, 26, 155-181.

\begin{abstract}
Financial difficulties are not an avoidable situation in the future for any company. This situation could emerge due to the combined effects of excessive financial leverage and greater competitiveness in markets. These factors are relevant when they are accompanied by economic crises or financial difficulties in the international economic situation, affecting as the headquarters and the countries in which the company has facilities and develops its activities. In this context, it is possible to evaluate the degree of resilience of companies using analysis of financial statements. A model based on this financial analysis that has been used since decades ago in predicting bankruptcy of companies is the "Z-score" model developed by Edward Altman. The Z-score, which can be applied mainly to companies traded on stock exchanges, has been used in the present study in order to estimate the probability of bankruptcy of the companies that comprise the Mexican Stock Exchange, at the end of the year 2015.
\end{abstract}

\section{Keywords}

Z-score, financial analysis, Mexican Stock Exchange. 


\section{De la Vega, J. (2016). Indicateur d'alerte rapide appliquée aux entreprises qui composent l'indice des prix et des taux à la Bourse mexicaine.Activos, 26, 155-181.}

\section{Résumé}

Plusieurs entreprises ont fait faillite dans le passé et beaucoup d'autres pourraient être dans la même situation à l'avenir. Cela peut être attribué aux effets combinés de l'augmentation de la compétitivité du marché et a excessif effet de levier financier. Ces facteurs sont plus pertinents lorsqu'ils sont accompagnés par des crises économiques ou des problèmes étrangers aux entreprises et qui affectent économiquement les pays où se développent leurs activités. La probabilité de survie des entreprises peut être déduite en utilisant l'analyse des états financiers. Il existe un modèle basé sur ces analyses et qui s'est révélé crédible pour prédire la faillite des entreprises deux ans avant que cela ne survienne; il s' agit du modèle du Z-Score, d'Edward Altman. Le Z-Score, qui s'applique de préférence aux entreprises du secteur de la fabrication et qui sont cotées en bourse, a été utilisé dans cette étude pour estimer la probabilité de faillite des entreprises qui composent l'indice des prix et des taux de la Bourse mexicaine à la fin de 2015.

\section{Mots-clés}

Z-Score, analyse financière, Bourse mexicaine.

\section{Introducción}

Las empresas que emiten deuda soportan siempre al menos un riesgo latente: que cuando la deuda venza no puedan pagarles a sus acreedores. Si esta situación adversa se materializa y el apalancamiento financiero es importante, entonces las empresas podrían incurrir en la quiebra o bancarrota (Brealey, Cooper y Kaplanis, 2012). 
Tanto los administradores como los accionistas, acreedores y empleados están preocupados por el buen desempeño financiero de las empresas y su saludable condición financiera. Los analistas coinciden en que tanto las aportaciones de los accionistas como los saldos a favor de los acreedores no estarán 100 \% garantizados en cualquier momento (Born, Payne, Scholar, Lin y Wen, 2014).

Debido a que una corporación es una entidad legal independiente, cuando fracasa en el pago de sus deudas, las entidades que le han suministrado dinero y sus acreedores en general estarán en posición de embargar los activos de la corporación en compensación por el incumplimiento o impago. Luego resulta trascendente reflexionar y desarrollar de forma verosímil la manera de predecir si una empresa podría incurrir en situación de impago en el corto y mediano plazo, con miras a tomar decisiones anticipadas y evitar esta situación (Berk, DeMarzo, Harford, Ford, Mollica y Finch, 2013).

Existen diversos tipos de análisis y modelos que identifican situaciones de turbulencia financiera en las empresas. Académicos europeos identificaron anticipadamente 139 firmas que registraron bancarrota durante el período de 1983 a 1994; para ello, utilizaron diversas variables de los estados financieros aplicando el complejo modelo Black-Scholes de valuación de opciones (Charitou y Trigeogis, 2002).

Para efectos de pronosticar la probabilidad de bancarrota también existe el modelo $\mathrm{O}$-Score, que, mediante el uso de un análisis logit- -técnica estadística desarrollada en análisis de mercados para valorar el grado de aceptación del cliente hacia un producto determinado- pronostica la probabilidad de bancarrota en un período de un año (Ohlson, 1980). A pesar de su existencia, dicho modelo no ha sido de uso extensivo y no se considera muy eficiente para estos efectos (Busso, DiNardo y McCrary, 2014).

Por otra parte, se destaca la existencia de un modelo en especial que, por su facilidad en pronosticar la probabilidad de quiebra o bancarrota, lo convierte en uno de los análisis más usados en los Estados Unidos para determinar la factible continuidad de una empresa, además de que sus 
resultados estadísticos son de fácil interpretación. El referido modelo se denomina marcador $Z$ de Altman o Altman's $Z$ - Score, y representa una alerta temprana que permite diagnosticar la probabilidad de que una empresa incurra en bancarrota en un futuro cercano e interpretar su nivel de salud financiera (Altman, 1968).

El presente estudio se centra en las empresas que al día $1 .^{\circ}$ de enero de 2015 conforman el índice de precios y cotizaciones de la Bolsa Mexicana de Valores, para alertar a las que poseen un perfil financiero de futuras dificultades financieras. Se requiere previamente la identificación cuantitativa de las empresas que poseen el mencionado perfil y luego identificar las posibles causas que refuerzan cualitativamente esta posibilidad en el mediano plazo.

\section{Marco teórico}

El análisis de estados financieros sirve para conocer el desempeño de una compañía en el pasado, así como para predecir su situación en el futuro. Algunos analistas que utilizan el análisis de estados financieros para interpretar razones financieras tienen preocupaciones e inquietudes específicas, como las siguientes (Gibson, 2012):

- Los auditores están interesados en juzgar si una empresa que manifiesta problemas financieros es candidata a continuar de la misma manera en el futuro.

- Los administradores están interesados en conocer los problemas financieros que encaran y la manera apropiada de aplicar una acción correctiva.

- Muchos prestamistas o proveedores de financiamiento emplean los modelos de marcadores de crédito para evaluar la solvencia y liquidez de los prestatarios o titulares de préstamos potenciales. La idea general es encontrar los factores que hagan posible que los prestamistas discriminen entre un riesgo crediticio bueno y uno malo. 
- Finalmente, los prestatarios quieren identificar los atributos del prestamista que puedan servir para predecir su incumplimiento o bancarrota y, por tanto, violentar la operación de crédito en un momento determinado.

En 1966, Altman desarrolló un modelo utilizando razones de estados financieros y análisis de discriminación múltiple para predecir la bancarrota en empresas públicamente negociadas del sector de manufactura o empresas que elaboran materias primas, productos semielaborados y productos terminados. Altman utilizó una muestra de sesenta y seis corporaciones, de las cuales treinta y tres, que corresponden al $50 \%$, habían incurrido en bancarrota en los últimos veinte años (Wijaya y Anantadjaya, 2014).

Altman calculó veintidós razones financieras para las sesenta y seis corporaciones referidas, pero haciendo hincapié en las empresas que más tarde se declararon en quiebra, utilizando estados financieros que fueron dados a conocer un año antes de la bancarrota. El objetivo fue seleccionar un pequeño número de esas razones financieras con la intención de poder diferenciar entre una empresa con probabilidades de ir a bancarrota y una empresa saludable.

Para realizar esta selección, Altman perfeccionó una técnica estadística de análisis de discriminación múltiple desarrollada inicialmente por Beaver (1966), utilizando para ello razones financieras que consideraban tanto el flujo de efectivo como el grado de apalancamiento de las empresas, entre otros indicadores. Lo atractivo en la técnica de Beaver era que muchas características podían ser combinadas dentro de un solo resultado, el cual, si era cercano a cero, implicaba bancarrota. Por lo tanto, Altman no fue el principal pionero en este análisis, dado que su aportación ha sido el perfeccionamiento de la técnica que Beaver impulsó para predecir bancarrota en las empresas públicas norteamericanas (Lee, 2014).

Para probar su modelo, Altman aplicó su fórmula desarrollada, que denominó como marcador $Z$, en grupos de empresas que habían incurrido en bancarrota y en grupos de empresas que no habían caído en dicha 
situación en los últimos años. Entre las compañías que habían incurrido en bancarrota, seleccionó aquellas que habían reportado pérdidas durante los tres años anteriores. Su propósito fue descubrir si el marcador $\mathrm{Z}$ podía distinguir entre compañías con problemas financieros y compañías con problemas financieros graves (Altman, Danovi y Falini, 2013).

Los resultados que arrojó el análisis fueron que cerca del $95 \%$ de las empresas que se habían declarado en bancarrota habían sido correctamente clasificadas por su modelo como empresas que poseían tales destinos, y aproximadamente el $80 \%$ de las empresas clasificadas como compañías que no iban a ir a bancarrota por su modelo efectivamente no incurrieron en tal situación.

Este modelo funciona como un previsor de bancarrota que combina cinco razones financieras, usando una ecuación que les asigna ciertos coeficientes para determinar la probabilidad de que una compañía manufacturera y pública incurra en bancarrota, en el entendido de que una empresa manufacturera es aquella que elabora materias primas, productos semiterminados y productos terminados. No obstante, este modelo no se limita al sector manufacturero, ya que se ha demostrado que su aplicación es verosímil, con algunas modificaciones, en firmas que no son del referido sector (Altman y Hotchkiss, 2005).

Las razones financieras utilizadas en el cálculo del marcador $\mathrm{Z}$ se basan en los siguientes siete rubros de los estados financieros, los cuales se conceptualizan a continuación (Vázquez y Díaz, 2013):

- Capital de trabajo neto. La Norma internacional de contabilidad $n .^{\circ} 1$ (NIC 1) establece que el capital de trabajo representa la diferencia entre los activos corrientes y los pasivos corrientes. La referida norma define los activos corrientes como aquellos que se esperan realizar o se tienen para su venta y consumo en el ciclo normal de explotación de la empresa, se mantienen o se espera transformar en efectivo o equivalentes dentro de un período de doce meses desde la fecha del balance, y se trata de efectivo u otro medio líquido equivalente cuya utilización no 
esté restringida. Por otra parte, la referida norma internacional de contabilidad define como pasivos corrientes las obligaciones que se espera liquidar en el curso normal de la explotación de la empresa y que deben liquidarse dentro de doce meses desde la fecha del balance.

- Activo total. La Norma internacional de contabilidad $n .^{\circ} 1$ señala que el activo total incluye tanto el activo corriente como el no corriente, en donde este último incluye los activos materiales o inmateriales, sean de explotación o financieros, ligados a la empresa a largo plazo. A pesar de lo anterior, la referida norma advierte que no está prohibido el uso de descripciones alternativas a las mencionadas, siempre que su significado sea análogo y quede claro.

- Utilidades antes de intereses e impuestos (UAII). Este concepto es identificado en la Norma internacional de contabilidad $n .{ }^{\circ} 1$ como resultado de explotación, el cual es definido como el margen bruto menos gastos de distribución, gastos de administración, gastos de explotación y considerando el efecto de otros ingresos de explotación.

- Valor de mercado del capital. En primera instancia, este valor está representado simplemente por el producto del precio de mercado de la acción por el número de acciones. No obstante, la Norma internacional de contabilidad $n .^{\circ} 1$ identifica este concepto con el nombre alternativo de patrimonio neto y lo define como el valor total de los siguientes conceptos: capital suscrito, prima de emisión, reservas de reevaluación, reservas legales y estatutarias, otras reservas, resultados diferidos y resultado del ejercicio.

- Valor en libros de la deuda. El Marco conceptual de las normas internacionales de contabilidad identifica los pasivos como sinónimo de este concepto. Los pasivos son considerados como aquellos rubros de los estados financieros que representan obligaciones de la empresa en el momento presente, resultando en compromisos o responsabilidades que pueden ser exigibles legalmente a consecuencia de la ejecución de un contrato o de una obligación de tipo legal. Finalmente, se aclara que 
dichas obligaciones o pasivos surgen además por la actividad normal de la empresa y por sus relaciones comerciales (Pacter, 2015).

- Ventas. Este concepto es identificado en el Marco conceptual de las normas internacionales de contabilidad como la suma de ingresos ordinarios y las ganancias. Los ingresos ordinarios surgen en el curso de las actividades ordinarias de la empresa y corresponden a una variada gama de denominaciones, tales como ventas, honorarios, intereses, dividendos, alquileres y regalías. Por su parte, son ganancias otras partidas que, siendo también ingresos, pueden o no surgir de las actividades ordinarias llevadas a cabo por la empresa.

- Utilidades retenidas acumuladas. Este concepto se define en la Norma internacional de contabilidad $n .^{\circ} 1$ como la suma algebraica de los resultados de ejercicios anteriores, en donde el resultado de cada uno de ellos se integró considerando los siguientes conceptos: ingresos ordinarios, gastos financieros, participación en el resultado del ejercicio en empresas asociadas y negocios conjuntos, y considerando el efecto del impuesto sobre las ganancias.

Una vez definidos los rubros de los estados financieros que toman parte en la integración del modelo de estudio que sirve para predecir de forma verosímil la bancarrota, se muestra a continuación la ecuación del marcador Z de Altman en su versión original, aplicable a las empresas manufactureras públicas (Hillier, Clacher, Ross, Westerfield y Jordan, 2014), modelo en el cual las razones financieras seleccionadas son multiplicadas por su determinado peso o ponderación y el resultado es sumado (tabla 1).

Tabla 1. Marcador $\mathrm{Z}$ de Altman (versión original)

\begin{tabular}{l|c|c}
\hline Sumatoria de: & & \\
\hline UAII/Total activo & $\times$ & 3.3 \\
\hline Ventas/Total activo & $\times$ & 1.0 \\
\hline Valor de mercado del capital/ Total pasivo & $\times$ & 0.6 \\
\hline Capital de trabajo/Total activo & $\times$ & 1.2 \\
\hline Utilidades retenidas acumuladas/Total activo & $\times$ & 1.4 \\
\hline
\end{tabular}

Fuente: Tomado de Hillier, Clacher, Ross, Westerfield y Jordan (2014). 
Un marcador $Z$ inferior a 1.81 indica que la empresa tiene $95 \%$ de probabilidad de caer en bancarrota en menos de dos años. No obstante, los resultados muestran que debe pensarse en el área entre 1.81 y 2.99 como un área gris. Por lo tanto, el modelo pronostica con seguridad bancarrota si Z es menor que o igual a 1.81 y la no bancarrota si $Z$ es mayor que o igual a 2.99. Altman manifiesta que las empresas en bancarrota y las empresas que no van a la bancarrota presentan perfiles financieros muy distintos desde un año antes de que ocurra esta debacle, siendo estos perfiles la clave del razonamiento tras el modelo del marcador Z. Por otra parte, y como se ha mencionado, el modelo original del marcador $Z$ requiere que las empresas sean de manufactura y con capital públicamente negociado.

No obstante, para hacer este modelo aplicable a las empresas privadas y públicas no manufactureras, a continuación (tabla 2) se muestra la posibilidad de emplear un marcador $\mathrm{Z}$ revisado.

Tabla 2. Marcador $\mathrm{Z}$ de Altman, versión revisada

\begin{tabular}{l|c|c}
\hline Sumatoria de: & & \\
\hline UAII/Total activo & $\times$ & 1.05 \\
\hline Valor de mercado del capital/Total pasivo & $\times$ & 6.72 \\
\hline Capital de trabajo/Total activo & $\times$ & 6.56 \\
\hline Utilidades retenidas acumuladas/Total activo & $\times$ & 3.26 \\
\hline
\end{tabular}

Fuente: Tomado de Hillier, Clacher, Ross, Westerfield y Jordan (2014).

Un marcador $Z$ revisado inferior a 1.23 indica que la empresa tiene 95 $\%$ de probabilidad de caer en bancarrota en menos de un año; si el marcador $Z$ revisado muestra valores entre 1.23 y 2.90 , debe establecerse este rango como un área gris. Por lo tanto, se predice bancarrota si el marcador $\mathrm{Z}$ revisado es menor que o igual a 1.23 y la no bancarrota si es mayor que o igual a 2.90 .

Finalmente, cabe aclarar que el marcador $\mathrm{Z}$ no predice con total exactitud si una compañía caerá formal o jurídicamente en una declaración de bancarrota en el corto y mediano plazo. Es, en cambio, una medida para identificar la similitud de una compañía con respecto a otras que se han 
declarado en bancarrota históricamente. Entonces, el marcador $\mathrm{Z}$ es una medida para conocer la salud financiera de la empresa y medir el riesgo de bancarrota económica, pero nunca es su finalidad predecir esta situación aseverando un destino fatal inevitable para la entidad (Altman, IwaniczDrozdowska, Laitinen y Suvas, 2016).

\section{Modelo de estudio}

Para realizar la predicción verosímil de bancarrota en las empresas que conforman el índice de precios y cotizaciones en la Bolsa Mexicana de Valores, el modelo de estudio del marcador $\mathrm{Z}$ en su versión original define su aritmética (tabla 3 ) de la siguiente forma:

Tabla 3. Modelo de estudio: marcador Z de Altman

\begin{tabular}{l|c|c}
\hline Sumatoria de: & & \\
\hline UAII/Total activo & $\times$ & 3.3 \\
\hline Ventas/Total activo & $\times$ & 1.0 \\
\hline Valor de mercado del capital/ Total pasivo & $\times$ & 0.6 \\
\hline Capital de trabajo/Total activo & $\times$ & 1.2 \\
\hline Utilidades retenidas acumuladas/Total activo & $\times$ & 1.4 \\
\hline
\end{tabular}

Fuente: Tomado de Hillier, Clacher, Ross, Westerfield y Jordan (2014).

Este modelo seleccionado permite determinar de forma sencilla la situación general que presenta una empresa en un momento de su historia, para ello utiliza ratios financieros claves. Al emplear este modelo es conveniente analizar la tendencia del comportamiento en el tiempo y las variaciones en el marcador Z, para tomar medidas correctivas en forma oportuna. El marcador $Z$ representa un interesante instrumento para evaluar las empresas y su salud financiera, $y$, aunque está lejos de la perfección, es un recurso valioso que puede ser complementado con otras técnicas de análisis fundamental para pronosticar quiebras o bancarrotas en las empresas.

En este punto, es importante discutir brevemente por qué utilizar el marcador $\mathrm{Z}$ y no otros para predecir de forma verosímil la probabilidad de bancarrota en una empresa pública: 
- El modelo O-Score enunciado anteriormente no está enfocado enteramente hacia indicadores financieros. Más bien está enfocado en la probabilidad de bancarrota desde el punto de vista de la mercadotecnia, en la que el grado de aceptación del producto por parte de los clientes es la variable fundamental.

- El modelo Beaver de predicción de bancarrota, desarrollado en 1966, finalmente ha sido perfeccionado por el marcador Z de Altman, siendo este último uno de los más difundidos en los libros de finanzas contemporáneos que abarcan en su contenido temas referentes a la predicción de bancarrota, quiebra o impago.

\section{Metodología}

Para el presente análisis se diseñó una investigación con las siguientes características:

- Tipo de investigación. No experimental, cuantitativa, utilizando información financiera después de ocurridos los hechos y mediante datos históricos para el año 2015 de los estados financieros de las empresas que conforman el índice de precios y cotizaciones en la Bolsa Mexicana de Valores.

- Fuentes de información. Se realizó una investigación documental, utilizando para ello fuentes de información secundaria de bases de datos de alta calidad y $100 \%$ verificables (Reuters, 2016).

- Extensión del estudio. Se realizó una investigación de corte transversal, utilizando información financiera del año más reciente disponible, el año 2015, con objeto de obtener resultados a corto plazo y que deberán ir cambiando año con año según la variación en los indicadores financieros establecidos en el modelo de estudio. Por lo tanto, se recolectaron datos en un tiempo único con el propósito de describir variables y analizar su incidencia e interpretación. 
- Nivel de medición y análisis de la información. Se realizó una investigación descriptiva; se calculó el marcador Z, normal y revisado, para todas las empresas que conforman el índice de precios y cotizaciones de la Bolsa Mexicana de Valores, determinando, por medio de este indicador, la posibilidad de que algunas de las referidas empresas incurran, de forma verosímil, en bancarrota en el corto y mediano plazo.

Para la realización de esta aplicación y análisis del marcador Z, se identifican a continuación (tabla 4) las empresas emisoras que conforman el índice de precios y cotizaciones en México (Bolsa Mexicana de Valores, 2015).

Tabla 4. Composición del índice de precios y cotizaciones de la Bolsa Mexicana de Valores

\begin{tabular}{|c|c|}
\hline EMISORA & SERIE \\
\hline $1 \mathrm{AC}$ & * \\
\hline 2 ALFA & A \\
\hline 3 ALSEA & * \\
\hline 4 AMX & $\mathrm{L}$ \\
\hline 5 ASUR & B \\
\hline 6 BIMBO & A \\
\hline 7 CÉMEX & $\mathrm{CPO}$ \\
\hline 8 ELEKTRA & * \\
\hline 9 FEMSA & UBD \\
\hline 10 GAP & $\mathrm{B}$ \\
\hline 11 GCARSO & $\mathrm{A} 1$ \\
\hline 12 GENTERA & * \\
\hline 13 GFINBUR & 0 \\
\hline 14 GFNORTE & $\mathrm{O}$ \\
\hline 15 GFREGIO & 0 \\
\hline 16 GMÉXICO & $\mathrm{B}$ \\
\hline 17 GRUMA & $\mathrm{B}$ \\
\hline 18 ICA & $\star$ \\
\hline 19 ICH & $\mathrm{B}$ \\
\hline 20 IENOVA & * \\
\hline 21 KIMBER & $\mathrm{A}$ \\
\hline $22 \quad$ KOF & $\mathrm{L}$ \\
\hline
\end{tabular}




\begin{tabular}{cl|c}
\hline \multicolumn{2}{c|}{ EMISORA } & SERIE \\
\hline 23 & LAB & $\mathrm{B}$ \\
\hline 24 & LA COMER & $\mathrm{UBC}$ \\
\hline 25 & LALA & $\mathrm{B}$ \\
\hline 26 & LIVEPOL & $\mathrm{C}-1$ \\
\hline 27 & MEXICHEM & $\star$ \\
\hline 28 & NEMAK & $\mathrm{A}$ \\
\hline 29 & OHLMEX & $\star$ \\
\hline 30 & OMA & $\mathrm{B}$ \\
\hline 31 & PEÑOLES & $\star$ \\
\hline 32 & PINFRA & $*$ \\
\hline 33 & SÁNMEX & $\mathrm{B}$ \\
\hline 34 & SIMEC & $\mathrm{B}$ \\
\hline 35 & SITES & $\mathrm{L}$ \\
\hline 36 & TLEVISA & CPO \\
\hline 37 & WÁLMEX & $\star$ \\
\hline
\end{tabular}

Fuente: Bolsa Mexicana de Valores (diciembre de 2015)

\section{Resultados obtenidos}

Después aplicar el modelo del marcador $\mathrm{Z}$ a las empresas que conforman el índice de precios y cotizaciones en la Bolsa Mexicana de Valores, y con fundamento en el marco teórico de la presente investigación, los resultados obtenidos se exponen a continuación.

Se identificaron las empresas que obtuvieron un marcador $Z$ por arriba de 2.99 y que son financieramente estables. Este grupo se conforma de once empresas que después de la aplicación del marcador $\mathrm{Z}$ obtuvieron resultados satisfactorios y entre las que destaca Infraestructura Energética Nova (IENOVA), con un marcador Z de 40.87 (tabla 5). 
Tabla 5. Empresas que cotizan en la Bolsa Mexicana de Valores sin probabilidad de incurrir en bancarrota

\begin{tabular}{l|c}
\hline EMISORA & MARCADOR Z (año 2015) \\
\hline IENOVA & 40.85 \\
\hline GMEXICO & 18.09 \\
\hline WÁLMEX & 12.84 \\
\hline MEXICHEM & 9.80 \\
\hline ASUR & 9.71 \\
\hline LALA & 9.46 \\
\hline PINFRA & 7.40 \\
\hline GAP & 7.22 \\
\hline SIMEC & 6.01 \\
\hline LIVEPOL & 5.75 \\
\hline GCARSO & 5.13 \\
\hline
\end{tabular}

Fuente: elaboración propia

De las emisoras anteriores, sus respectivas descripciones son las siguientes, de tal manera que permitan identificar los sectores con un sano desempeño en la economía mexicana durante el año 2015:

- IENOVA. Infraestructura Energética Nova S. A. B. de C. V. es una empresa con sede en México que se desarrolla en el sector de servicios públicos. Sus actividades se organizan en dos segmentos: gas y electricidad. En el segmento de gas, la compañía está involucrada en el desarrollo y la operación de ductos de gas natural y gas licuado de petróleo o GLP, así como en el almacenamiento, transporte, la distribución y comercialización de gas natural y GLP, todo esto en varios estados del país, incluyendo Baja California, Sonora, Chihuahua, Durango, Tamaulipas, Nuevo León y Jalisco. Además, posee y opera una unidad de almacenamiento y regasificación de gas natural licuado o GNL en Baja California, que se utiliza en la importación de GNL. En el segmento de electricidad, la empresa desarrolla la gestión y operación de una central eléctrica de gas natural que incluye dos turbinas de gas y una turbina de vapor. 
- GMÉXICO. Grupo México S. A. B. de C. V. (Grupo México) desarrolla actividades de la industria minero-metalúrgica, así como la exploración, la explotación y el beneficio de productos minerales metálicos y no metálicos; desarrolla también el servicio multimodal de mercancías por medio de ferrocarril. Sus filiales incluyen las subsidiarias Americas Mining Corporation (AMC), Southern Copper Corporation (SCC) en México y Perú y Asarco en Estados Unidos. La división de Transporte de la Sociedad está representada por sus filiales Infraestructura y Transportes México S. A. de CV (ITM) y FM Rail Holding S. A. de CV (FM Rail Holding). La división de infraestructura de la compañía está representada por su filial México Proyectos y Desarrollos S. A. de C. V. (MPD).

- WÁLMEX. Wal-Mart de México S. A. B. de C. V. (WALMEX) Opera en tiendas de autoservicio. Los segmentos de la compañía incluyen operaciones en México y América Central. En México, la compañía opera por medio del concepto de autoservicio, que incluye la operación de tiendas de descuento, hipermercados, tiendas de venta al por mayor, supermercados y otros conceptos que incluyen los grandes almacenes y las operaciones inmobiliarias con terceros. En América Central opera en tiendas de descuento, supermercados, hipermercados, tiendas de almacenes y tiendas de venta al por mayor en Costa Rica, Guatemala, Honduras, Nicaragua y El Salvador. Ofrece productos en categorías tales como alimentos, artículos de consumo, mercancía y ropa en general. Ofrece sus productos bajo varias marcas, incluyendo Bodega Aurrerá Express, Walmart, Sams Club, Superama, Suburbia y Medimart Farmacias. La compañía desarrolla también el negocio de comercio electrónico en México, operando en tres sitios de Internet: walmart. com.mx, sams.com.mx y superama.com.mx

- MEXICHEM. Mexichem S. A. B. de C. V. es una empresa con sede en México que se dedica principalmente a la transformación de productos químicos y petroquímicos en productos y soluciones que se utilizan en los sectores industriales de la construcción y la agricultura. Sus actividades se estructuran en tres cadenas de producción: cadena cloro-vinilo, en la que se desarrolla la extracción de cloro para la 
producción de cloruro de polivinilo (PVC) y soda cáustica destinada a la fabricación de jabones, champús y detergentes; cadena flúor, centrada en la extracción de fluorita y su transformación en ácido de grado metalúrgico y ácido fluorhídrico; y cadena de soluciones integrales, especializada en la producción de sistemas de tuberías de PVC, conexiones y accesorios de plástico, así como en geosistemas tales como geotextiles y geodrenajes, entre otros. La compañía está presente en todo el continente americano, así como en Europa y Asia; en 2013 adquirió activos de las empresas PolyOne Corp. y Wavin NV, empresas fabricantes de tuberías y accesorios de plástico.

- LALA. Grupo LALA S. A. B. de C. V. es una empresa con sede en México que se dedica principalmente a la fabricación de productos lácteos. La cartera de productos de la compañía incluye leche light, entera, sin lactosa, descremada, leche con calcio extra, yogures, queso, mantequilla, margarina y los probióticos, entre otros; también ofrece jugos y postres de gelatina. Además, la compañía es propietaria de Lala Institute (Instituto Lala), que apoya y lleva a cabo la investigación en nutrición, provee de orientación alimentaria y promueve la vida sana, entre otros servicios.

- PINFRA. Promotora y Operadora de Infraestructura S. A. B. de C. V. opera en quince concesiones viales y en un puerto. La empresa desarrolla la construcción, la operación, el mantenimiento, el financiamiento y la promoción de proyectos carreteros, portuarios, ferroviarios y aeroportuarios, así como la construcción y operación de estacionamientos, plantas de tratamiento de aguas residuales y agua potable, también operaciones de prestación de servicios de alcantarillado, recolección y barrido de basura. Los principales giros de negocio en los cuales participa se dividen en manufactura, concesiones y construcción.

- GAP. Grupo Aeroportuario del Sureste S. A. B. de C. V. opera en los nueve aeropuertos más importantes del sureste de México: Cancún, Cozumel, Huatulco, Mérida, Minatitlán, Oaxaca, Tapachula, Veracruz 
y Villahermosa. En todos los aeropuertos del Grupo se alcanza la cifra récord de 19.3 millones de pasajeros a partir del año 2012 y posteriores. En el año 2010, ASUR festejó sus primeros 10 años de cotizar en la Bolsa Mexicana de Valores y en el NYSE o New York Stock Exchange.

- SIMEC. Grupo Simec, S. A. B. de C. V. fue constituido en agosto de 1990. Es un fabricante y distribuidor de productos de acero con operaciones de producción y comercialización en los Estados Unidos, México y Canadá. Sus productos se utilizan en una amplia gama de aplicaciones, incluyendo ejes, cigüeñales y ejes para automóviles y camiones ligeros, así como para el desarrollo de máquinas y herramientas para el sector de la construcción, principalmente en el mercado de la construcción no residencial. En los Estados Unidos y México, Simec posee y opera en doce plantas, con una capacidad instalada para fabricar acero 4.5 millones de toneladas. Sus principales plantas de producción se localizan en Mexicali, Guadalajara, Tlaxcala y San Luis Potosí.

- LIVEPOL. El Puerto de Liverpool S. A. B. de C. V. es una empresa con sede en México que se dedica principalmente al sector minorista. Las actividades de la compañía están estructuradas en tres áreas de negocio: división al por menor, que opera en una red de numerosos grandes almacenes Liverpool y Fábricas de Francia, así como un número de tiendas libres de impuestos y tiendas especializadas; división inmobiliaria, incluyendo la gestión y explotación de centros comerciales; y la división de crédito, con la emisión de tarjetas de crédito VISA-marca bajo el nombre de Liverpool, ofreciendo créditos de consumo y servicios de financiación de ventas.

- GCARSO. Grupo Carso S. A. B. de C. V. es un conglomerado diversificado con actividades principales en toda América Latina. Los segmentos de la compañía incluyen la venta al por menor de artículos industriales de infraestructura y construcción. Opera en varios sectores por medio de sus filiales Grupo Sanborns, Grupo Condumex y Carso Infraestructura y Construcción. Su sector Grupo Sanborns opera en diversos puntos de venta al por menor, incluidos 
los grandes almacenes y tiendas de venta al por menor con diversos artículos, incluyendo de electrónica y entretenimiento, y opera también en restaurantes. Su sector Grupo Condumex tiene una cartera de productos y servicios centrados en las industrias de telecomunicaciones, construcción, electricidad, automotriz y minera. Finalmente, su sector Carso Infraestructura y Construcción ofrece una gama de productos y servicios tales como plataformas de petróleo; perforación de pozos de petróleo, gas y geotérmicos; construcción de infraestructura tales como centros comerciales, oficinas, viviendas, carreteras, presas y plantas de tratamiento de agua.

En adición, se identificaron las emisoras que obtuvieron un marcador $\mathrm{Z}$ dentro del rango de 1.81 y 2.99 (tabla 6), las cuales son clasificadas como empresas dentro del área gris y que, si bien no son empresas con altas probabilidades de caer en bancarrota, es verosímil afirmar que este grupo sí está conformado por empresas que de no mejorar su situación financiera $-\mathrm{y}$ teniendo en consideración el entorno económico adverso y lleno de turbulencias e inestabilidades, con un escenario tanto de bajo crecimiento como de una inflación a la alza en el corto y mediano plazo - presentan probabilidades más latentes de incurrir en problemas financieros más graves a consecuencia de poseer un relevante grado de apalancamiento financiero. Entre las empresas que se destacan y que se encuentran en el área gris, se observan las siguientes:

Tabla 6. Empresas que cotizan en la Bolsa Mexicana de Valores con baja probabilidad de incurrir en bancarrota

\begin{tabular}{c|c}
\hline EMISORA & MARCADOR Z (año 2015) \\
\hline BIMBO & 2.75 \\
\hline AC & 2.75 \\
\hline ALSEA & 2.71 \\
\hline LAB & 2.69 \\
\hline NEMAK & 2.44 \\
\hline KOF & 2.23 \\
\hline ALFA & 2.14 \\
\hline
\end{tabular}

Fuente: elaboración propia 
Posteriormente, se identificaron las empresas que obtuvieron un marcador $\mathrm{Z}$ menor que 1.81, indicador de que la situación financiera de estas empresas es muy perfectible o está sujeta a un área de mejora, pero en ningún caso se asume probabilidad alguna de que incurran en problemas de insolvencia, dado que para tal aseveración es necesario un análisis más profundo que el marcador $Z$, solo es una referencia del período de auge o dificultad que enfrenta el sector en el que se desarrolla cada una de estas empresas (tabla 7):

Tabla 7. Empresas que cotizan en la Bolsa Mexicana de Valores con marcador $\mathrm{Z}$ inferior a 1.81

\begin{tabular}{c|c}
\hline EMISORA & $\begin{array}{c}\text { MARCADOR Z } \\
\text { (año 2015) }\end{array}$ \\
\hline TLEVISA & 1,72 \\
\hline AMX & 1,55 \\
\hline OHLMEX & 1,30 \\
\hline ELEKTRA & 1,23 \\
\hline CÉMEX & 0,82 \\
\hline SITES & 0,73 \\
\hline GFINBUR & 0,70 \\
\hline GFREGIO & 0,53 \\
\hline GFNORTE & 0,41 \\
\hline SÁNMEX & 0,32 \\
\hline ICA & 0,01 \\
\hline
\end{tabular}

Fuente: elaboración propia

De las emisoras anteriores, sus respectivas descripciones son las siguientes, de tal manera que permitan identificar los sectores que enfrentan una situación en mayor desafío o circunstancias menos ventajosas en la economía mexicana durante el año 2015:

- TELEVISA: Grupo Televisa S. A. B. es una empresa de medios de comunicación en la industria global de entretenimiento. La compañía opera en cuatro segmentos de negocios: contenidos, televisión, 
telecomunicaciones y otros negocios. Opera en cuatro canales de difusión en la Ciudad de México y se ha afiliado a diversas estaciones en todo el país. Produce canales de televisión de paga con canales nacionales e internacionales en toda América Latina, Estados Unidos, Canadá, Europa y Asia Pacífico. Exporta sus programas y formatos a las redes de televisión de todo el mundo. Al 31 de diciembre de 2014, la compañía exportó 87.143 horas de programación a 83 países, con excepción de Estados Unidos. La compañía es también una editorial de revistas en español, con una circulación anual de aproximadamente 117 millones de revistas, que publican 182 títulos en aproximadamente 21 países.

- AMX: América Móvil, S. A. B. de C. V. fue constituida el 13 de octubre de 2000. La compañía ofrece servicios de telecomunicaciones en aproximadamente 30 países. Sus servicios de telecomunicaciones incluyen servicios de voz de telefonía fija y móvil, servicios de datos inalámbricos y fijos, acceso a Internet y televisión de paga, así como venta de accesorios, equipos y otros servicios relacionados con el sector de las telecomunicaciones. Los segmentos de la compañía incluyen geográficamente a México, Brasil, Colombia, región Andina en Suramérica, América Central, el Caribe, Estados Unidos y Europa. En México, el segmento móvil de la compañía incluye las operaciones de su filial Radiomóvil Dipsa S. A. de C. V., mejor conocida como Telcel. A su vez, el segmento de telefonía fija en México se presenta con su filial Teléfonos de México S. A. B. de C. V. o Telmex. En Brasil, sus subsidiarias son Claro S. A. (Claro Brasil), Americel S. A. (Americel), Embratel TVSAT Telecomunicações S. A. (Claro TV) y Star One S. A. (Star One). El segmento de Colombia incluye las operaciones de las subsidiarias Comunicación Celular S. A. (Comcel) y Telmex Colombia S. A. (Telmex Colombia).

- OHLMEX: OHL México S. A. B. de C. V. es una empresa con sede en México dedicada a la gestión del transporte y a la infraestructura aeroportuaria. Cuenta con una amplia cartera de concesiones, compuesta por seis autopistas de peaje que interconectan las zonas urbanas del Distrito Federal y los estados de México y Puebla. 
Además, también está implicada en la gestión integral del Aeropuerto Internacional de Toluca.

- ELEKTRA: Grupo Elektra S. A. B. de C. V. es una empresa de servicios financieros y de comercio especializado. La compañía opera en alrededor 6.000 puntos de venta en México, Estados Unidos, Guatemala, Honduras, Perú, Panamá y El Salvador. La compañía se dirige a los consumidores en la base de la pirámide socioeconómica en América Latina. La empresa ofrece bienes en los segmentos de electrónica, electrodomésticos, muebles, transporte, teléfonos, computadoras, así como el servicio de transferencias electrónicas de dinero y garantías extendidas, entre otros productos y servicios.

- CÉMEX: CÉMEX, S. A. B. de C. V. (CÉMEX) fue constituida el 11 de junio de 1920. Es una empresa que principalmente se desarrolla en la producción, distribución, comercialización y venta de cemento, concreto premezclado y otros materiales de construcción en todo el mundo, y que ofrece servicios relacionados con la construcción a clientes y comunidades en más de 50 países de todo el mundo. La compañía opera en los segmentos geográficos de México, Estados Unidos, norte de Europa, Mediterráneo, América del Sur, el Caribe y Asia. Sus instalaciones de producción de cemento se encuentran en México, Estados Unidos, España, Egipto, Alemania, Colombia, Filipinas, Polonia, República Dominicana, Reino Unido, Croacia, Panamá, Letonia, Puerto Rico, Tailandia, Costa Rica y Nicaragua.

- SITES: Telesites S. A. B. de C. V. es una empresa con sede en México que participa en el campo de las telecomunicaciones inalámbricas. La compañía se centra en la operación y el mantenimiento de las instalaciones de transmisión que proporcionan comunicaciones directas a través de ondas de radio. Su gama de soluciones se divide en dos segmentos: alquiler de infraestructura y construcción de estaciones de transmisión. Uno de los principales clientes de la compañía es Radiomóvil Dipsa S. A. de C. V. (Telcel). 
- GFINBUR: Grupo Financiero Inbursa S. A. B. de C. V., con sede en México, participa por medio de sus subsidiarias en el sector financiero. Las principales actividades de la compañía están estructuradas en cuatro líneas de negocio: banca comercial, gestión de activos, seguros y banca de inversión. La compañía está involucrada en la prestación de servicios tales como tarjetas de crédito y débito, préstamos personales, hipotecas, depósitos a plazo, fondos de inversión, servicios de corretaje, financiación de las empresas, pólizas de seguros y fondos de pensiones, entre otros servicios financieros.

- GFREGIO: BanRegio Grupo Financiero S. A. B. de C. V. es una institución financiera con sede en México que participa en el sector bancario. Por medio de sus subsidiarias, ofrece una variedad de productos y servicios financieros, tales como la provisión de créditos corporativos con gran atención a las pequeñas y medianas empresas (PYME), préstamos personales, hipotecas, tarjetas de crédito y débito, seguros personales y corporativos, así como gestión de inversiones, entre otros servicios.

- GFNORTE: Grupo Financiero Banorte S. A. B. de C. V. es una compañía con sede en México que por medio de sus subsidiarias participa en el sector financiero. La compañía está involucrada en la prestación de múltiples servicios bancarios, incluidos los servicios de ahorro, la negociación de valores, préstamos hipotecarios, la administración de fondos de pensiones, pólizas de seguros, intermediación de crédito y otros servicios financieros dirigidos a empresas y particulares, así como a las pequeñas y medianas empresas (PYME). La compañía está activa en el país y en el extranjero. En octubre de 2013, Assicurazioni Generali $S p A$ vendió el $49 \%$ de su capital accionario a las empresas mexicanas Seguros Banorte Generali y Pensiones Banorte Generali, del Grupo Financiero Banorte S. A. B. de C. V.

- SÁNMEX: Grupo Financiero Santander México S. A. B. de C. V., anteriormente Santander Financial Group México S. A. B. de C. V. o Grupo Financiero Santander S. A. B. de C. V., es una institución 
financiera con sede en México. La empresa se dedica principalmente a la prestación de servicios bancarios, el corretaje de valores, servicios de asesoramiento financiero, así como a otras actividades de inversión relacionadas, atendiendo a clientes individuales y corporativos. Sus principales filiales son Banco Santander (México) S. A., Casa de Bolsa Santander S. A. de C. V. y Zurich Santander Seguros México S. A.

- ICA: Empresas ICA S. A. B. de C. V. fue constituida el 25 de julio de 1979. Por medio de sus subsidiarias, la compañía se dedica a brindar servicios para el sector de la construcción y actividades relacionadas, incluyendo la construcción de instalaciones de infraestructura, así como la construcción industrial, urbana y de vivienda, tanto para el sector público como para el privado de México. ICA opera en cinco segmentos: construcción civil, construcción industrial, concesiones, aeropuertos y otros servicios corporativos. ICA también está involucrada en la construcción, el mantenimiento y la operación de carreteras, puentes y túneles otorgados en concesión por el Gobierno Federal y los gobiernos extranjeros en régimen de concesión. Por medio de sus subsidiarias y filiales, la compañía también administra y opera en aeropuertos. Además, ICA se dedica a la prestación de servicios relacionados con la minería, tales como la exploración y explotación de yacimientos, el transporte y otras actividades relacionadas con la minería.

\section{Conclusiones}

Se afirma que sí es posible identificar de manera verosímil la probabilidad de que una empresa pública enfrente dificultades o situaciones de salud financiera por medio del cálculo de marcador $Z$, sin que esto represente en absoluto un predictor de insolvencia en el corto o mediano plazo.

Por lo tanto, en el presente estudio se aplicó el modelo del marcador Z de Altman a las empresas listadas en el índice de precios y cotizaciones de la Bolsa Mexicana de Valores, para determinar la salud financiera de las entidades en el mediano plazo. 
Cabe señalar que después de haber realizado el análisis del marcador $\mathrm{Z}$ de Altman a las empresas públicas o cotizadas mexicanas, los resultados encontrados fueron interesantes, puesto que varias de las referidas empresas que se dedican a o que reciben gran parte de sus ingresos de sectores como el de la construcción e inmobiliario, así como las del sector financiero y de telecomunicaciones, se encontraron entre algunas de las compañías que fueron calificadas como en situación de mayor desafío en el contexto actual económico, tanto a nivel nacional (México) como internacional. Esto se debe a su perfectible desempeño financiero en términos de poseer alto apalancamiento, aunado a ser empresas que atraviesan y están resintiendo los efectos de una crisis económica y financiera que tuvo sus orígenes en el año 2008 y que hasta el presente no se ha logrado superar a nivel mundial, crisis que está repercutiendo en estos sectores en el mundo y que coincidió con el resultado de niveles inferiores a 1.81 en la aplicación del marcador Z.

La intención de este marcador no es pronosticar con precisión cuándo una empresa se declarará en bancarrota; su finalidad es dar a conocer de manera un tanto general, pero verosímil, mediante un indicador, las condiciones financieras con las que opera la empresa y si es financieramente saludable, con miras a tomar decisiones anticipadas y evitar llevar a la compañía a la bancarrota en el mediano plazo.

Una alerta temprana es una buena manera de denominar a este instrumento financiero debido a su capacidad de influir en la toma de decisiones preventivas si el resultado es poco satisfactorio.

Entonces, se concluye que este tipo de análisis es un instrumento útil para los administradores de empresas o ejecutivos financieros en la tarea de evaluar la situación actual e histórica de las empresas. En el presente análisis se demuestran la utilidad y el aprovechamiento de este modelo, indicador que podría significar una gran diferencia para la toma de decisiones eficientes en las empresas. 


\section{Referencias}

Altman, E. I. (1968). Financial ratios, discriminant analysis and the prediction of corporate bankruptcy. The Journal of Finance, 23(4), 589-609. DOI: $10.2307 / 2978933$

Altman, E. I., Danovi, A. y Falini, A. (2013). Z-score models' application to Italian companies subject to extraordinary administration. Journal of Applied Finance (Formerly Financial Practice and Education), 23(1), 128-137.

Altman, E. I., Iwanicz-Drozdowska, M., Laitinen, E. K. y Suvas, A. (2016). Financial distress prediction in an international context: A review and empirical analysis of Altman's Z-Score Model. Journal of International Financial Management \& Accounting. DOI: 10.1111/jifm.12053

Altman, E. I. y Hotchkiss, E. (2005). Corporate financial distress and bankruptcy: Predict and avoid bankruptcy (3. ${ }^{\text {a }}$ ed.). U. S. A.: McGraw-Hill.

Beaver, W. H. (1966). Financial ratios as predictors of failure. Journal of Accounting Research, 4, 71-111. DOI: 10.2307/2490171

Berk, J., DeMarzo, P., Harford, J., Ford, G., Mollica, V. y Finch, N. (2012). Fundamentals of corporate finance. Pearson Higher Education AU.

Bolsa Mexicana de Valores. (2015). Muestra del índice de precios y cotizaciones. Recuperado de http://www.bmv.com.mx/

Born, P., Payne, H., Scholar, C. H. M. E., Lin, H. A. y Wen, M. M. (2014). Cashflow risk management in the insurance industry: A dynamic factor modeling approach. Recuperado de: http://sfm.finance.nsysu.edu.tw/php/Papers/ CompletePaper/057-1691085211.pdf

Busso, M., DiNardo, J. y McCrary, J. (2014). New evidence on the finite sample properties of propensity score reweighting and matching estimators. Review of Economics and Statistics, 96(5), 885-897. DOI: 10.1162/REST_a_00431

Brealey, R. A., Cooper, I. A. y Kaplanis, E. (2012). International propagation of the credit crisis: Lessons for bank regulation. Journal of Applied Corporate Finance, 24(4), 36-45.

Charitou, A. y Trigeogis, L. (2000). Option-based bankruptcy prediction. Recuperado de goo.gl/lAkFBc.

Gibson, C. H. (2012). Financial statement analysis. South-Western. 
Hillier, D., Clacher, I., Ross, S., Westerfield, R. y Jordan, B. (2014). Fundamentals of corporate finance (2. ${ }^{\text {a }}$ edición europea). Londres: McGraw Hill.

Lee, M. C. (2014). Business bankruptcy prediction based on survival analysis approach. International Journal of Computer Science \& Information Technology, 6(2), 103-119. DOI:10.5121/ijcsit.2014.6207

Ohlson, J. (1980). Financial ratios and the probabilistic prediction of bankruptcy. Journal of Accounting Research, 19(1), 109-301. DOI: 10.2307/2490395

Pacter, P. (2015). IFRS as global standards: A pocket guide. Londres: IFRS Foundation. Recuperado de goo.gl/z1XY72

Reuters. (2016). Business \& Financial News, Breaking US \& International News. Reuters. Recuperado de http://www.reuters.com/

Vázquez, N. y Díaz, M. (2013). Normas internacionales de información financiera (NIIF-IFRS). Barcelona: Profit Editorial.

Wijaya, S. V. y Anantadjaya, S. P. (2014). Bankruptcy Prediction Model: An industrial study in Indonesian publicly-listed firms during 1999-2010. RIBER: Review of Integrative Business \& Economics Research, 3(1), 13-41. Recuperado de goo.gl/IPqECg 